OPEN ACCESS

University of Dundee

\title{
Adherence to once-daily and twice-daily direct acting antiviral therapy for hepatitis C infection among people with recent injection drug use or current opioid agonist therapy
}

Cunningham, Evan B.; Hajarizadeh, Behzad; Amin, Janaki; Litwin, Alain H.; Gane, Edward; Cooper, Curtis

Published in:

Clinical Infectious Diseases

DOI:

$10.1093 / \mathrm{cid} / \mathrm{ciz} 1089$

Publication date:

2020

Document Version

Peer reviewed version

Link to publication in Discovery Research Portal

Citation for published version (APA):

Cunningham, E. B., Hajarizadeh, B., Amin, J., Litwin, A. H., Gane, E., Cooper, C., Lacombe, K., Hellard, M., Read, P., Powis, J., Dalgard, O., Bruneau, J., Matthews, G. V., Feld, J. J., Dillon, J. F., Shaw, D., Bruggmann, P., Conway, B., Fraser, C., ... Grebely, J. (2020). Adherence to once-daily and twice-daily direct acting antiviral therapy for hepatitis $\mathrm{C}$ infection among people with recent injection drug use or current opioid agonist therapy. Clinical Infectious Diseases, 71(7), e115-e124. https://doi.org/10.1093/cid/ciz1089

\section{General rights}

Copyright and moral rights for the publications made accessible in Discovery Research Portal are retained by the authors and/or other copyright owners and it is a condition of accessing publications that users recognise and abide by the legal requirements associated with these rights.

- Users may download and print one copy of any publication from Discovery Research Portal for the purpose of private study or research. - You may not further distribute the material or use it for any profit-making activity or commercial gain.

- You may freely distribute the URL identifying the publication in the public portal.

Take down policy

If you believe that this document breaches copyright please contact us providing details, and we will remove access to the work immediately and investigate your claim. 


\section{Adherence to once-daily and twice-daily direct acting antiviral therapy for hepatitis $\mathrm{C}$}

infection among people with recent injection drug use or current opioid agonist therapy

Evan B Cunningham ${ }^{1}$, Behzad Hajarizadeh ${ }^{1}$, Janaki Amin ${ }^{1,2}$, Alain H Litwin ${ }^{3,4,5}$, Edward Gane $^{6}$, Curtis Cooper ${ }^{7}$, Karine Lacombe ${ }^{8}$, Margaret Hellard ${ }^{9,10}$, Phillip Read ${ }^{11}$, Jeff Powis ${ }^{12}$, Olav Dalgard ${ }^{13,14}$, Julie Bruneau ${ }^{15}$, Gail V Matthews ${ }^{1,16}$, Jordan J Feld ${ }^{17}$, John F Dillon ${ }^{18}$, David Shaw $^{19}$, Philip Bruggmann ${ }^{20}$, Brian Conway ${ }^{21}$, Chris Fraser²2, Philippa Marks ${ }^{1}$, Gregory J Dore ${ }^{1,16}$, Jason Grebely ${ }^{1}$

${ }^{1}$ The Kirby Institute, UNSW Sydney, Sydney, Australia, ${ }^{2}$ Faculty of Medicine and Health Sciences, Macquarie University, Sydney, Australia, ${ }^{3}$ University of South Carolina-Greenville, Greenville, SC, USA, ${ }^{4}$ Clemson University, Greenville, SC, USA, ${ }^{5}$ Prisma Health, Greenville, SC, USA, ${ }^{6}$ Auckland City Hospital, Auckland, New Zealand, ${ }^{7}$ Ottawa Hospital Research Institute, Ottawa, Canada, ${ }^{8}$ Inserm UMR-S1136, Sorbonne Université, Hôpital Saint-Antoine, AP-HP, Paris, France, ${ }^{9}$ The Burnet Institute, Melbourne, Australia, ${ }^{10}$ Department of Infectious Disease, The Alfred Hospital, Melbourne, Australia, ${ }^{11}$ Kirketon Road Centre, Sydney, Australia, ${ }^{12}$ South Riverdale Community Health Centre, Toronto, Canada, ${ }^{13}$ Akershus University Hospital, Oslo, Norway, ${ }^{14}$ Institute of Clinical Medicine, University of Oslo, Oslo, Norway, ${ }^{15}$ Centre Hospitalier de l'Université de Montréal, Canada,

${ }^{16}$ St Vincent's Hospital, Sydney, Australia, ${ }^{17}$ Toronto General Hospital, Toronto, ${ }^{18}$ Ninewells Hospital and Medical School, University of Dundee, Dundee, United Kingdom, ${ }^{19}$ Royal Adelaide Hospital, Adelaide, Australia, ${ }^{20}$ Arud Centres for Addiction Medicine, Zurich, Switzerland, ${ }^{21}$ Vancouver Infectious Diseases Center, Vancouver, Canada, ${ }^{22}$ Coolaid Community Health Centre, Victoria, Canada 


\section{Corresponding Author:}

Dr. Evan Cunningham

Postdoctoral fellow

The Kirby Institute

UNSW Sydney

Wallace Wurth Building

UNSW NSW 2052 Australia

E-mail: ecunningham@kirby.unsw.edu.au

Summary: Adherence to once- and twice-daily DAA therapy was high but reduced among those on twice-daily therapy. Despite risk factors for non-adherence (stimulant injecting, unstable housing, later treatment time), non-adherence did not significantly affect treatment outcomes suggesting considerable forgiveness to non-adherence. 


\begin{abstract}
Background: This study investigated treatment adherence and associated factors among people with recent injecting drug use or current opioid agonist therapy (OAT) and compared once-daily to twice-daily DAA therapy.
\end{abstract}

Methods: SIMPLIFY and D3FEAT are international, multicentre studies which recruited participants with recent injecting drug use (previous six months; SIMPLIFY, D3FEAT) or current OAT (D3FEAT) between March 2016 and February 2017 in eight countries. Participants received sofosbuvir/velpatasvir (once-daily; SIMPLIFY) or paritaprevir/ritonavir/ombitasvir, dasabuvir (twice-daily) \pm ribavirin (D3FEAT) for 12 weeks administered in electronic blister-packs. We evaluated overall adherence (proportion of prescribed doses taken) and non-adherence ( $<90 \%$ adherent) with comparisons between dosing patterns.

Results: Of 190 participants who commenced treatment, 184 (97\%) completed treatment. Median adherence was $92 \%$ with higher adherence among those receiving once-daily vs. twice-daily therapy $(94 \%$ vs. $87 \%, P=0.005)$. Overall, $40 \%$ of participants $(n=76)$ were considered non-adherent ( $<90 \%$ adherent). Recent stimulant injecting (odds ratio [OR] 2.48, 95\% confidence interval [CI] 1.28-4.82), unstable housing (OR 2.18, 95\% CI 1.01-4.70), and receiving twice-daily dosing (OR 2.81, 95\% CI 1.47-5.36) were associated with nonadherence. Adherence decreased over the course of therapy SVR was high in non-adherent $(89 \%)$ and adherent populations $(95 \%, \mathrm{P}=0.174)$ with no difference in SVR between those who did and did not miss at least seven consecutive doses (92\% vs 93\%, $P=0.897)$. 
Conclusion: This study demonstrated high adherence to once- and twice-daily HCV DAA therapy among people with recent injecting drug use or were currently receiving OAT. The levels of non-adherence described did not impact treatment outcomes, suggesting forgiveness to non-adherence.

Keywords: HCV, treatment, PWID, drug use, injecting drug users, adherence, OAT 


\section{Introduction}

Although direct-acting antiviral (DAA) therapy is effective among people who inject drugs (PWID) [1], little is known about adherence, including factors associated with non-adherence and the impact of adherence on sustained virologic response (SVR). In many settings, there remains resistance among some clinicians to provide HCV treatment for PWID on the basis that poor adherence may compromise treatment outcomes [2-4].

Studies from the interferon-era have demonstrated that treatment completion and adherence are comparable between people with and without recent injecting drug use $[5,6]$. In the DAA era, a small number of studies have demonstrated high adherence to DAA therapy among people with recent injecting drug use [6-8] and people receiving opioid agonist therapy (OAT) [9-12]. The majority of studies evaluating adherence among people receiving OAT or people with recent injecting drug use have used imprecise methods for measuring adherence, have heterogenous definitions of recent injecting drug use, are often single-centre, and are limited by small sample sizes. No study has compared once-daily and twice-daily DAA therapy.

SIMPLIFY and D3FEAT are two international, multicentre phase-4 trials of HCV DAA treatment that used electronic blister packs to assess adherence among people with recent (last six months) injecting drug use or currently receiving OAT $[13,14]$. The aims of this analysis were to evaluate adherence to DAA therapy and associated factors and to compare adherence between those receiving once-daily and twice-daily therapy. 


\section{Methods}

\section{Study design and participants}

In two international, multicentre, open-label phase 4 trials (SIMPLIFY and D3FEAT), participants were enrolled at 25 sites in Australia (seven sites), Canada (six sites), France (two sites), New Zealand (two sites), Norway (two sites), Switzerland (four sites), the United Kingdom (one site), and the United States (one site) (SIMPLIFY, ClinicalTrials.gov: NCT02336139; and D3FEAT, ClinicalTrials.gov: NCT02498015). These sites included four drug and alcohol clinics, one private practice, 17 hospital clinics, and three community clinics.

Participants were 18 years of age or older, had chronic HCV genotypes 1-6, were HCV treatment-naïve, and had injected drugs in the last six months (self-reported at enrolment; SIMPLIFY and D3FEAT) or were currently receiving OAT (D3FEAT; Figure 1). Participants with HIV or decompensated liver disease were excluded. All participants provided written informed consent before study procedures started.

\section{Procedures}

The study design of the SIMPLIFY and D3FEAT studies have previously been reported [13, 14]. In SIMPLIFY, patients received one co-formulated sofosbuvir/velpatasvir tablet oncedaily for 12 weeks. In D3FEAT, patients with HCV genotype 1a received two co-formulated paritaprevir/ritonavir/ombitasvir tablets once-daily, and one dasabuvir tablet twice-daily for 12 weeks. Participants with genotype 1a also received weight-based ribavirin twice-daily.

Participants in D3FEAT received ribavirin in pill bottles. All other study drugs were dosed weekly in electronic blister packs (Information Mediary Corporation, Ottawa, Canada) that 
recorded the date and time each dose was removed. In SIMPLIFY, the blister packs contained one tablet per day in a single blister. In D3FEAT, the blister packs contained three tablets in individual blisters for the morning dose and one tablet in a single blister for the evening dose (Figure 2). Participants received AUS\$10 (or equivalent) to return each blister pack. Adherence was also measured by counting remaining pills in the returned blister packs (clinical pill count) and through four-weekly self-reported adherence questionnaires.

Participants completed a self-administered questionnaire on a tablet computer at enrolment, at treatment commencement and every fourth week during treatment. The questionnaires collected information on demographics, drug and alcohol use, and injecting risk behaviours. Stable housing was defined as living in a rented or privately-owned house or flat with all other housing categories defined as unstable housing. Hazardous alcohol consumption was evaluated using the AUDIT-C [15].

\section{Outcomes}

The primary endpoint for this analysis was non-adherence to DAA therapy, defined as receiving the correct dosing on fewer than $90 \%$ of the intended days of treatment as measured by electronic blister pack. Correct dosing was at least one dose (one tablet) per day in SIMPLIFY and at least two doses (four tablets) per day in D3FEAT. Ribavirin dosing was not included in analyses. Where more than the expected number of doses was removed in one day adherence was recorded as $100 \%$ for the day. In the case of damaged blister packs $(n=7)$ or participants removing pills without breaking the senor grid $(n=2)$, clinical pill count was used. 
Overall adherence was a secondary endpoint, calculated by dividing the number of doses removed from the blister pack (to a maximum of one per day in SIMPLIFY and two per day in D3FEAT) by the number of expected doses (84 doses in SIMPLIFY and 168 for D3FEAT). Weekly adherence was calculated assuming all pills removed in a week were taken correctly to a maximum of $100 \%$ adherence in each week. Overall weekly adherence was calculated as the mean of the adherence for each treatment week. Self-reported adherence to therapy was calculated by dividing the number of pills taken by the expected number of pills.

\section{Statistical analysis}

Participants with $<90 \%$ (non-adherence) and $\geq 90 \%$ adherence were compared using Pearson's chi-square test. Logistic regression was used to assess predictors of non-adherence. Hypothesised predictors included age (stratified by median), sex, education, hazardous alcohol consumption, current OAT, past month injecting drug use (any, heroin, cocaine, amphetamine, stimulant [cocaine or amphetamine]), frequency of injecting drug use, and region of residence (North America, Australia/New Zealand, or Europe). All variables with $\mathrm{p}<0.20$ in the unadjusted analyses were considered for multivariate logistic regression models using a backward stepwise approach.

The impact of time on treatment was assessed using generalised estimating equation (GEE) analyses by including day of treatment as a factor in the model adjusted for age, sex, current OAT, heroin injecting, stimulant injecting, unstable housing, and hazardous alcohol consumption. As dosing pattern (once- vs twice-daily dosing) was determined to be a potential effect modifier, GEE analyses were done stratified by dosing pattern. 
Statistically significant differences were assessed at $\mathrm{p}<0.05 ; \mathrm{p}$ values are two-sided. All analyses were performed using the statistical package Stata v14.1 (College Station, TX, United States). 


\section{Results}

Baseline characteristics

190 participants initiated DAA therapy (SIMPLIFY $n=103$, D3FEAT $n=87$ ). The baseline behavioural and demographic characteristics are shown in Table 1. The median age was 48 years, $74 \%$ were male, and $49 \%$ reported a high school education or greater.

At baseline, participants included those who had injected drugs in the last six months and were not on OAT $(33 \%, \mathrm{n}=63)$, those with injecting in the last six months on OAT $(47 \%$, $\mathrm{n}=90)$, and those without injecting in the last six months on OAT $(19 \%, \mathrm{n}=37$; D3FEAT only; Figure 1). Sixty-one percent $(\mathrm{n}=115)$ had injected drugs in the last month. The drugs most commonly injected in the month prior to commencement of therapy were heroin (44\%) and amphetamines (24\%; Table 1). In the D3FEAT study, 90\% $(n=78)$ were receiving ribavirin.

Differences between those receiving once- (SIMPLIFY) compared to twice-daily (D3FEAT) therapy are presented in Table 1. Participants receiving twice-daily therapy were more likely to be receiving OAT at baseline and less likely to have injected any drugs in the last month or be residing outside Australia/New Zealand. Among people with injecting drug use in the past month, there was no significant difference in injecting frequency, or the types of drugs injected between those receiving once- or twice-daily therapy. The only difference between participants who reported injecting in the last six months at enrolment (SIMPLIFY and D3FEAT) and those who did not (D3FEAT) was region of residence with participants with current injecting were more likely to reside in Europe (Supplementary Table 1). 


\section{Treatment completion and adherence}

$184(97 \%)$ of 190 participants completed treatment as defined by attending the end of treatment study visit (Table 2). Reasons for not completing treatment were loss to follow-up $(n=3)$, incarceration $(n=1)$, physician-directed discontinuation $(n=1)$, and death due to overdose $(n=1)$.

Overall adherence as measured by blister pack, was 92\% (interquartile range [IQR], 81-98\%; Table 2 and Figure 3) and was higher among those receiving once-daily compared to twicedaily therapy ( $94 \%$ vs $87 \%, \mathrm{p}=0.005$; Figure 4). Adherence was higher when measured by self-report (99\% [IQR, 97-100\%]) and weekly-assessed blister pack adherence (98\% [IQR 94-100\%]). Patient-reported reasons for non-adherence by blister pack assessment were available in 175 instances over the course of therapy and included "forgot" ( $\mathrm{n}=104,59 \%)$, “inaccessible at time of dose" (n=31,18\%), "side effects" (n=17,10\%), "lost" (n=10,6\%), and "other" $(\mathrm{n}=13,7 \%)$.

By daily blister pack measurement, $90 \%(\mathrm{n}=171)$ of participants did not take all prescribed doses on at least one day of treatment and $48 \%$ of participants did not all prescribed doses on between one and eight days. Episodes of non-adherence lasted for no more than one consecutive day in $42 \%$ of participants. Twenty-five participants (13\%) had an episode of non-adherence for $\geq 7$ consecutive days.

\section{Baseline predictors of non-adherence}

The proportion of participants with $<90 \%$ blister pack adherence (non-adherence) stratified by key behavioural and demographic characteristics is shown in Table 3. In adjusted analyses, factors independently associated with non-adherence included unstable housing 
(aOR 2.18, 95\% CI 1.01-4.70), stimulant injecting (last month; aOR 2.48, 95\% CI 1.28-4.82), and twice-daily dosing (aOR 2.81, 95\% CI 1.47-5.36).

In a sensitivity analysis excluding participants from D3FEAT who did not report injecting in the last six months at enrolment, factors independently associated with non-adherence included stimulant injecting (last month; aOR 2.32, 95\% CI 1.16-4.65) and twice-daily dosing (aOR 3.26, 95\% CI 1.57-6.79; Supplementary Table 2).

\section{Change in adherence over the course of therapy}

The change in adherence over the course of therapy stratified by dosing pattern is shown in Figure 5. In GEE analyses, later treatment period was associated with increased odds of nonadherence (per week; aOR 1.08, 95\% CI 1.06-1.09). When models were stratified by prescribed dosing pattern, this effect remained for both once-daily dosing (per week; aOR 1.08, 95\% CI 1.06-1.11) and twice-daily dosing (per week; aOR 1.08, 95\% CI 1.06-1.10).

\section{Impact of DAA adherence on SVR}

SVR by intent-to-treat was 93\% (176 of 190). Among participants who did not achieve SVR, the reasons for not achieving SVR included virologic failure $(n=3)$, reinfection $(n=1)$, loss to follow-up on treatment $(n=6)$, lost to follow-up following treatment $(n=2)$, and death $(n=2)$. All three participants with virologic failure were receiving twice-daily paritaprevir/ritonavir/ombitasvir, dasabuvir (individual daily blister pack adherence was 99\%, $98 \%$ and $86 \%$ ).

SVR was lower among those not adherent to therapy, although not significant (89\% vs 95\%, $\mathrm{p}=0.174)$ and similar when those who were lost to follow-up were excluded (99\% vs $97 \%$ 
$\mathrm{p}=0.579$ ). There was no difference in SVR among those with and without any missed doses (92\% vs $95 \%, P=0.711$ ) or among those who did and did not miss at least seven consecutive doses (92\% vs 93\%, $P=0.897)$. Of the 25 participants with an episode of non-adherence for at least seven consecutive days, 21 (84\%) completed treatment with no virologic failures. Eleven participants had an overall adherence of less than 50\%, among whom six achieved SVR with no virologic failures. The remaining five participants were lost to follow-up. 


\section{Discussion}

This study evaluated adherence to HCV DAA therapy and associated factors and compared adherence between those receiving sofosbuvir/velpatasvir (once-daily) and paritaprevir/ritonavir/ombitasvir, dasabuvir with or without ribavirin (twice-daily) therapy in people with injecting drug use in the last six months or receiving OAT. High adherence to therapy was observed, although adherence declined during treatment. Adherence was lower among people receiving twice-daily therapy. Unstable housing, stimulant injecting, and receiving twice-daily therapy were associated with non-adherence. Adherence, missed doses during therapy, and extended non-adherent episodes ( $\geq$ seven days) did not impact SVR, suggesting forgiveness to non-adherence with these two regimens. These data are important to inform clinical guidelines, clinical management, and health policy, particularly in settings where restrictions for the reimbursement of DAA therapy for PWID are in place.

The high median adherence (92\%) observed in this study is consistent with other studies among people with recent injecting drug use and people receiving OAT [7, 8, 16-21]. Previous studies have been limited by their adherence assessment methodologies (self-report or clinical pill count) and by small sample sizes. In this study, adherence to once-daily was higher compared to twice-daily therapy ( $94 \%$ vs $87 \%$ ). This finding is novel in the context of HCV DAA therapy, consistent with studies of HIV therapy demonstrating higher adherence to once-daily regimens [22-25]. These data highlight the importance of simplified dosing to optimize adherence among PWID and people receiving OAT.

A decline in adherence was observed during treatment, consistent with previous studies [19, 21]. The use of electronic blister packs for adherence monitoring was a major strength of this study, allowing for detailed and accurate adherence measurement over time, providing a more 
precise estimate of the effect of time on non-adherence. It is interesting that similar declines in adherence were observed irrespective of dosing pattern (once-daily vs. twice-daily). While there has been an interest in exploring shorter durations of DAA therapy, it is not clear whether there would be a similar 'forgiveness' to non-adherence with shorter durations of therapy. Further research is needed to evaluate the impact of non-adherence on SVR in the context of short-duration DAA therapy.

In addition to dosing pattern, recent stimulant injecting and unstable housing were associated with non-adherence. Although studies have demonstrated that recent injecting drug use is associated with reduced adherence, most studies have lacked the power to evaluate the effect of specific types of drugs on adherence $[5,6,19,21]$. The association between stimulant use and adherence may be of concern given the increasing prevalence of stimulant use reported in many countries globally [26]. Unstable housing was also independently associated with nonadherence. While homelessness has been shown to be associated with treatment failure [27] our finding of an association between unstable housing and non-adherence is novel and consistent with a systematic review demonstrating poorer adherence to HIV therapy among unstably housed populations [28]. Despite these factors impacting adherence, there was no significant impact on treatment outcome.

The limited impact of adherence on SVR was an encouraging finding. Among those who did not achieve an SVR, the primary reason for treatment failure was loss to follow-up during or following treatment. Of the three people who completed therapy and had virologic failure, adherence was high (99\%, 98\% and 86\%). Among those with adherence below 50\% (n=11), six achieved SVR with no recorded virologic failures. This included successful therapy among a participant with adherence of only $25 \%$. Despite the observed non-adherence and 
risk factors for non-adherence, these data highlight that the two HCV DAA regimens examined in this study have a considerable degree of forgiveness to non-adherence and support the inclusion this population in $\mathrm{HCV}$ treatment programs.

This study had some limitations. Although the method of adherence monitoring was precise, the blister packs required accurate and correct usage. For example, when more than the expected number of pills were removed on a given day, adherence was restricted to $100 \%$. If the additional pills removed were taken correctly on subsequent days, then the adherence recorded would underestimate the participant's true adherence. Alternatively, weekly adherence, which assumes all pills removed in a given week were taken correctly, likely overestimates a participant's true adherence. Therefore, a participant's true adherence likely lies somewhere between daily- and weekly-assessed blister pack adherence. Despite these concerns, blister pack measurement of adherence remains a more robust method of measuring adherence compared to clinical pill count or self-report [29].

Another limitation is that data for this analysis were combined from two separate clinical trials with different inclusion criteria (SIMPLIFY: injecting drug use in the last six months; D3FEAT injecting drug use in the last six months or receiving OAT); however, D3FEAT still recruited a high proportion of people with injecting drug use in the last six months. In sensitivity analyses excluding the participants from D3FEAT who did not report injecting in the last six months at enrolment, stimulant injecting in the last month and twice-daily therapy remained associated with non-adherence. Further, characteristics of the study populations were similar, likely due to the use of the same recruitment network for study enrolment, and any remaining differences were controlled for in adjusted analyses. Lastly, participants from 
D3FEAT who were receiving twice-daily therapy represented a less marginalized population, despite having poorer adherence.

The results of this study cannot necessarily be generalised to all populations of people who inject drugs and people receiving OAT. While the international nature of these data enhances the generalisability, participants likely represent a somewhat selected population who were engaged with health services and were not co-infected with HIV. Further, participants were treated in clinics which may have been more experienced in HCV treatment in these populations and the lack of randomisation could have resulted in unmeasured confounding due to, for example, the decision by study sites to include/exclude particular patients in the trials.

Lastly, adherence to therapy was likely enhanced by weekly contact with healthcare providers to return used blister packs and obtain subsequent doses, and the blister pack itself may have indirectly acted as an adherence support tool. Furthermore, although the incentive received for the return of the blister pack was not linked to the measured adherence, this incentive may have indirectly encouraged greater adherence. Despite these limitations, these data present a robust analysis of treatment adherence in a high-risk population of people with injecting drug use in the last six months and people receiving OAT.

Overall, adherence was high in this study. Different patterns of non-adherence did not impact SVR, suggesting a degree of forgiveness to non-adherence with the regimens of once-daily sofosbuvir/velpatasvir and twice-daily paritaprevir/ritonavir/ombitasvir, dasabuvir with or without ribavirin. Further research is needed to evaluate the impact of adherence on SVR in the context of shorter durations of DAA therapy. Taken together, these data support DAA 
therapy among people with recent injecting drug use and people receiving OAT. These data are important to inform clinical guidelines and improve clinical management of $\mathrm{HCV}$ infection among people with recent injecting drug use. Further, these data provide key information to support the removal of restrictions for the reimbursement of HCV DAA therapy for people with recent drug/alcohol use that are still in place in some settings globally $[30,31]$. 


\section{Acknowledgements}

The authors thank the study participants for their contribution to the research, as well as current and past researchers and staff.

\section{Declaration of Interests}

JG reports grants and personal fees from AbbVie, Cepheid, Gilead Sciences, and Merck. OD reports grants from Gilead Sciences during this study and grants from Gilead Sciences, Merck, and AbbVie, and advisory board fees from Abbvie and MSD. PB reports grants and personal fees from AbbVie, Bristol-Myers Squibb, Gilead Sciences, Mundipharma, and Merck Sharp \& Dohme. MH reports grants from Gilead Sciences, Bristol-Myers Squibb, and AbbVie. JBr reports consultant fees from Gilead Sciences and Merck. AHL reports grants and personal fees from Gilead Sciences, Merck, and personal fees from Abbvie. GVM reports grants and personal fees from Gilead Sciences and grants and personal fees from AbbVie. JP reports grants and personal fees from Janssen and Genetech. CC reports grants and personal fees from Gilead Sciences, Merck, and Abbvie. JJF reports grants and personal fees from AbbVie, Merck, Gilead Sciences, Roche, Wako/Fujifilm, and Janssen, personal fees from Contravir, and grants from Abbott and Enanta. CF reports grants and non-financial support from Kirby Institute, Abbvie, and Gilead Sciences during this study and grants from Gilead Sciences, ViiV HealthCare, Abbvie, and Merck, outside the submitted work. GJD reports grants, personal fees, and non-financial support from AbbVie, Merck, Bristol-Myers Squibb, and Roche; grants and personal fees from Janssen; personal fees and non-financial support from Gilead Sciences; and personal fees from GlaxoSmithKline and Abbott Diagnostics. PR

reports fees for educational talks from Gilead Sciences, Merck Sharp \& Dohme, and AbbVie, and is on the advisory board for Merck Sharp \& Dohme. BC reports grants, personal fees, and non-financial support from Gilead Sciences, Merck, AbbVie, Indivior, and ViiV. EG 
reports personal fees from being a Clinical Advisor for Gilead Sciences, Merck, Janssen, and AbbVie, and personal fees from Gilead Sciences Speaker Bureau and AbbVie Speaker Bureau. JFD reports grants from Abbvie during the course of the study, grants and personal fees from Gilead, Merck Sharp \& Dohme, Janssen, and AbbVie. BH reports grants from AbbVie and Gilead Sciences. DS reports clinical trial nurse support from Kirby Institute, during the study. KL reports advisory board fees and travel grants from Abbvie, MSD, and Gilead. All other authors declare no competing interests. 


\section{References}

[1] Hajarizadeh B, Cunningham EB, Reid H, Law M, Dore GJ, Grebely J. Direct-acting antiviral treatment for hepatitis $\mathrm{C}$ among people who use or inject drugs: a systematic review and meta-analysis. Lancet Gastroenterol Hepatol 2018.

[2] Barua S, Greenwald R, Grebely J, Dore GJ, Swan T, Taylor LE. Restrictions for medicaid reimbursement of sofosbuvir for the treatment of hepatitis c virus infection in the united states. Annals of Internal Medicine 2015;163:215-223.

[3] Marshall AD, Saeed S, Barrett L, Cooper CL, Treloar C, Bruneau J, et al. Restrictions for reimbursement of direct-acting antiviral treatment for hepatitis $\mathrm{C}$ virus infection in Canada: a descriptive study. CMAJ Open 2016;4:E605-E614.

[4] Ooka K, Connolly JJ, Lim JK. Medicaid Reimbursement for Oral Direct Antiviral Agents for the Treatment of Chronic Hepatitis C. Am J Gastroenterol 2017;112:828-832.

[5] Aspinall EJ, Corson S, Doyle JS, Grebely J, Hutchinson SJ, Dore GJ, et al. Treatment of Hepatitis C Virus Infection Among People Who Are Actively Injecting Drugs: A Systematic Review and Meta-analysis. Clinical Infectious Diseases 2013;57:S80-S89.

[6] Cunningham EB, Hajarizadeh B, Dalgard O, Amin J, Hellard M, Foster GR, et al. Adherence to response-guided pegylated interferon and ribavirin for people who inject drugs with hepatitis $C$ virus genotype 2/3 infection: the ACTIVATE study. Bmc Infectious Diseases 2017;17.

[7] Read P, Gilliver R, Kearley J, Lothian R, Cunningham EB, Chronister KJ, et al. Treatment Adherence and Support for People who Inject Drugs Taking Direct Acting Antiviral Therapy for Hepatitis C Infection. Journal of Viral Hepatitis 2018.

[8] Cunningham EB, Amin J, Feld JJ, Bruneau J, Dalgard O, Powis J, et al. Adherence to sofosbuvir and velpatasvir among people with chronic HCV infection and recent injection drug use: The SIMPLIFY study. International Journal of Drug Policy 2018;62:14-23. 
[9] Dore GJ, Altice F, Litwin AH, Dalgard O, Gane EJ, Shibolet O, et al. ElbasvirGrazoprevir to Treat Hepatitis C Virus Infection in Persons Receiving Opioid Agonist TherapyA Randomized TrialElbasvir-Grazoprevir in Persons With HCV Receiving OAT. Annals of internal medicine 2016;165:625-634.

[10] Grebely J, Mauss S, Brown A, Bronowicki J-P, Puoti M, Wyles D, et al. Efficacy and safety of ledipasvir/sofosbuvir with and without ribavirin in patients with chronic HCV genotype 1 infection receiving opioid substitution therapy: Analysis of Phase 3 ION trials. Clinical Infectious Diseases 2016;63:1405-1411.

[11] Martinello M, Hajarizadeh B, Grebely J, Dore GJ, Matthews GV. HCV Cure and Reinfection Among People With HIV/HCV Coinfection and People Who Inject Drugs. Curr Hiv-Aids Rep 2017;14:110-121.

[12] Lalezari J, Sullivan JG, Varunok P, Galen E, Kowdley KV, Rustgi V, et al. Ombitasvir/paritaprevir/r and dasabuvir plus ribavirin in HCV genotype 1-infected patients on methadone or buprenorphine. Journal of Hepatology 2015;63:364-369.

[13] Grebely J, Dalgard O, Conway B, Cunningham EB, Bruggmann P, Hajarizadeh B, et al. Efficacy and safety of sofosbuvir/velpatasvir in people with chronic hepatitis $\mathrm{C}$ viris infection and recent injecting drug use: the SIMPLIFY study. The lancet Gastroenterology \& hepatology 2017.

[14] Grebely J, Conway B, Cunningham EB, Fraser C, Moriggia A, Gane E, et al. Paritaprevir, ritonavir, ombitasvir, and dasabuvir with and without ribavirin in people with HCV genotype 1 and recent injecting drug use or receiving opioid substitution therapy. International Journal of Drug Policy 2018;Accepted.

[15] Bush K, Kivlahan DR, McDonell MB, Fihn SD, Bradley KA. The AUDIT alcohol consumption questions (AUDIT-C): an effective brief screening test for problem drinking. 
Ambulatory Care Quality Improvement Project (ACQUIP). Alcohol Use Disorders Identification Test. Archives of Internal Medicine 1998;158:1789-1795.

[16] Bielen R, Moreno C, Van Vlierberghe H, Bourgeois S, Mulkay J-P, Vanwolleghem T, et al. Belgian experience with direct acting antivirals in people who inject drugs. Drug and Alcohol Dependence 2017;177:214-220.

[17] Dore G, Altice F, Litwin AH, Dalgard O, Gane EJ, Shibolet O, et al. C-EDGE COSTAR: Efficacy of Grazoprevir and Elbasvir in Persons who Inject Drugs (PWID) Receiving Opioid Agonist Therapy. Hepatology 2015;62:227a-228a.

[18] Grebely J, Flamm S, Zeuzem S, Hyland R, Chang P, Ding X, et al. On-Treatment Illicit Drug Use Did Not Impact Treatment Outcome during Therapy with LedipasvirSofosbuvir with or without Ribavirin in the Phase 3 Ion-1 Study. Journal of Hepatology 2016;64:S776-S777.

[19] Litwin A, Agyemang L, Akiyama M, Heo M, Wong J, Soloway I, et al. High rates of sustained virological response in people who inject drugs treated with all-oral direct acting antiviral regimens. 5th International Symposium on Hepatitis Care in Substance Users, Oslo, Norway 2016.

[20] Mason K, Dodd Z, Guyton M, Tookey P, Lettner B, Matelski J, et al. Understanding real-world adherence in the directly acting antiviral era: A prospective evaluation of adherence among people with a history of drug use at a community-based program in Toronto, Canada. International Journal of Drug Policy 2017.

[21] Petersen T, Townsend K, Gordon LA, Sidharthan S, Silk R, Nelson A, et al. High adherence to all-oral directly acting antiviral HCV therapy among an inner-city patient population in a phase 2a study. Hepatology International 2016;10:310-319.

[22] Mbuagbaw L, Sivaramalingam B, Navarro T, Hobson N, Keepanasseril A, Wilczynski NJ, et al. Interventions for Enhancing Adherence to Antiretroviral Therapy 
(ART): A Systematic Review of High Quality Studies. Aids Patient Care and Stds 2015;29:248-266.

[23] Cooper V, Horne R, Gellaitry G, Vrijens B, Lange AC, Fisher M, et al. The Impact of Once-Nightly Versus Twice-Daily Dosing and Baseline Beliefs About HAART on Adherence to Efavirenz-Based HAART Over 48 Weeks: The NOCTE Study. Jaids-J Acq Imm Def 2010;53:369-377.

[24] Maitland D, Jackson A, Osorio J, Mandalia S, Gazzard BG, Moyle GJ, et al. Switching from twice-daily abacavir and lamivudine to the once-daily fixed-dose combination tablet of abacavir and lamivudine improves patient adherence and satisfaction with therapy. Hiv Medicine 2008;9:667-672.

[25] Parienti JJ, Bangsberg DR, Verdon R, Gardner EM. Better Adherence with OnceDaily Antiretroviral Regimens: A Meta-Analysis. Clinical Infectious Diseases 2009;48:484488.

[26] UNODC. World Drug Report 2019. Vienna; 2019.

[27] Gottfredsson M, Love T, Fridriksdottir RH, Tyrfingsson T, Runarsdottir V, Hansdottir I, et al. Is homelessness the biggest hurdle to treatment success in the management of HCV in the era of direct acting antivirals? Results from the TraP HepC nationwide treatment initiative in Iceland. ILC 2019 -Journal of Hepatology 2019;70:PS-072.

[28] Aidala AA, Wilson MG, Shubert V, Gogolishvili D, Globerman J, Rueda S, et al. Housing Status, Medical Care, and Health Outcomes Among People Living With HIV/AIDS: A Systematic Review. American Journal of Public Health 2016;106:95-95.

[29] Lam WY, Fresco P. Medication Adherence Measures: An Overview. Biomed Res Int 2015 . 
[30] Marshall AD, Cunningham EB, Nielsen S, Aghemo A, Alho H, Backmund M, et al. Restrictions for reimbursement of interferon-free direct-acting antiviral drugs for HCV infection in Europe. Lancet Gastroenterol Hepatol 2018;3:125-133.

[31] Marshall AD, Pawlotsky JM, Lazarus JV, Aghemo A, Dore GJ, Grebely J. The removal of DAA restrictions in Europe - One step closer to eliminating $\mathrm{HCV}$ as a major public health threat. J Hepatol 2018;69:1188-1196. 
Table 1: Participant characteristics at baseline overall and stratified by study

\begin{tabular}{|c|c|c|c|c|}
\hline & $\begin{array}{l}\text { Overall } \\
(n=190) \\
n(\%)\end{array}$ & $\begin{array}{l}\text { SIMPLIFY } \\
\text { (once-daily) } \\
(\mathbf{n}=103) \\
\mathrm{n}(\%) \\
\end{array}$ & $\begin{array}{l}\text { D3FEAT } \\
\text { (twice-daily) } \\
(\mathrm{n}=87) \\
\mathrm{n}(\%) \\
\end{array}$ & $\mathbf{P}$ \\
\hline Age, median (IQR) & $48(41-53)$ & $48(41-53)$ & $48(43-54)$ & 0.727 \\
\hline Male sex & $141(74)$ & $74(72)$ & $67(77)$ & 0.417 \\
\hline High school or greater education & $93(49)$ & $50(49)$ & $41(49)$ & 0.971 \\
\hline Unstable housing & $37(20)$ & $24(23)$ & $13(16)$ & 0.195 \\
\hline Hazardous alcohol consumption ${ }^{a}$ & $97(51)$ & $18(17)$ & $10(12)$ & 0.274 \\
\hline Opioid substitution treatment (current) & $158(83)$ & $58(56)$ & $62(73)$ & 0.018 \\
\hline OST and recent injecting (past month) & & & & $<0.001$ \\
\hline No OST, no recent injecting & $21(11)$ & $12(12)$ & $9(11)$ & \\
\hline No OST, recent injecting & $47(25)$ & $33(32)$ & $14(17)$ & \\
\hline OST, no recent injecting & $52(28)$ & $15(15)$ & $37(45)$ & \\
\hline OST, recent injecting & $68(36)$ & $43(42)$ & $23(28)$ & \\
\hline Study site distribution & & & & 0.003 \\
\hline Australia/New Zealand & $61(32)$ & $43(42)$ & $18(21)$ & \\
\hline North America & $78(41)$ & $40(39)$ & $38(44)$ & \\
\hline Europe & $51(27)$ & $20(19)$ & $31(36)$ & \\
\hline Any injecting drug use in the last month & $115(61)$ & $76(74)$ & $39(46)$ & $<0.001$ \\
\hline Injecting drug use >daily in the last month ${ }^{b}$ & $40(35)$ & $27(36)$ & $13(33)$ & 0.815 \\
\hline \multicolumn{5}{|l|}{ Drugs injected in the last month ${ }^{b}$} \\
\hline Heroin & $77(67)$ & $55(72)$ & $22(59)$ & 0.167 \\
\hline Cocaine & $21(18)$ & $12(16)$ & $9(24)$ & 0.274 \\
\hline Amphetamines & $42(37)$ & $27(36)$ & $15(41)$ & 0.605 \\
\hline
\end{tabular}

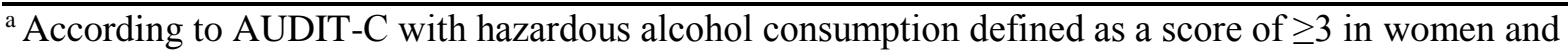
$\geq 4$ in men

${ }^{\mathrm{b}}$ Among those who reported injecting in the last month. 
Table 2: Measures of treatment completion and adherence stratified by dosing pattern.

\begin{tabular}{lccc}
\hline Variable & $\begin{array}{c}\text { Overall } \\
(\mathbf{n = 1 9 0 )} \\
\mathbf{n}(\%)\end{array}$ & $\begin{array}{c}\text { Once-daily } \\
(\mathbf{n = 1 0 3}) \\
\mathbf{n}(\mathbf{\%})\end{array}$ & $\begin{array}{c}\text { Twice-daily } \\
(\mathbf{n = 8 7}) \\
\mathbf{n}(\mathbf{\%})\end{array}$ \\
\hline Treatment completion & $184(97)$ & $100(97)$ & $84(97)$ \\
Number of days non-adherent to therapy, & & & \\
n (\%) & & & \\
$\quad$ None (100\% adherent) & $19(10)$ & $12(12)$ & $7(8)$ \\
$1-4(95-<100 \%$ adherent) & $56(29)$ & $36(35)$ & $20(23)$ \\
$5-8(90-<95 \%$ adherent) & $35(18)$ & $20(19)$ & $15(17)$ \\
$9-17(80-<90 \%$ adherent) & $34(18)$ & $17(17)$ & $17(20)$ \\
$\geq 18(<80 \%$ adherent) & $46(24)$ & $18(17)$ & $28(32)$ \\
Overall adherence (\% [95\% CI]) & & & \\
Patient report & $99(97-100)$ & $99(98-100)$ & $99(96-100)$ \\
Blister pack, weekly & $98(94-100)$ & $98(94-100)$ & $98(93-99)$ \\
Blister pack, daily & $92(81-98)$ & $94(88-98)$ & $88(75-96)$ \\
Longest episode of non-adherence (days) & & & \\
1 & $80(42)$ & $44(43)$ & $36(41)$ \\
2 & $39(21)$ & $19(18)$ & $20(23)$ \\
3 & $8(4)$ & $3(3)$ & $5(6)$ \\
4 & $11(6)$ & $9(9)$ & $2(2)$ \\
5 & $5(3)$ & $2(2)$ & $3(3)$ \\
6 & $3(2)$ & $3(3)$ & $0(0)$ \\
$\geq 7$ & $25(13)$ & $11(11)$ & $14(16)$ \\
\hline
\end{tabular}


Table 3: Logistic regression of factors associated with nonadherence $(<90 \%)$

\begin{tabular}{|c|c|c|c|c|c|c|}
\hline & $\begin{array}{l}\text { DAA } \\
\text { adherence of } \\
\geq 90 \%(\% ; \\
n=114)\end{array}$ & $\begin{array}{l}\text { DAA adherence } \\
\text { of }<90 \%(\% ; \\
n=76)\end{array}$ & Unadjusted OR & $\mathbf{P}$ & Adjusted OR & $\mathbf{P}$ \\
\hline \multicolumn{7}{|l|}{ Age (years) } \\
\hline$<48$ & $53(55)$ & $44(45)$ & 1.00 & - & & \\
\hline$>48$ & $61(66)$ & $32(34)$ & $0.63(0.35-1.13)$ & 0.124 & & \\
\hline \multicolumn{7}{|l|}{ Gender } \\
\hline Male & $81(57)$ & $60(43)$ & 1.00 & - & & \\
\hline Female & $33(67)$ & $16(33)$ & $0.65(0.33-1.30)$ & 0.225 & & \\
\hline \multicolumn{7}{|l|}{ Education } \\
\hline$<$ High school & $60(63)$ & $36(38)$ & 1.00 & - & & \\
\hline $\begin{array}{l}\text { High school or } \\
\text { greater }\end{array}$ & $53(58)$ & $38(42)$ & $0.97(0.85-1.10)$ & 0.606 & & \\
\hline \multicolumn{7}{|l|}{ Housing } \\
\hline Stable & $96(64)$ & $53(36)$ & 1.00 & & & \\
\hline Unstable & $17(46)$ & $20(54)$ & $2.13(1.03-4.41)$ & 0.042 & $2.18(1.01-4.70)$ & 0.046 \\
\hline \multicolumn{7}{|c|}{ Hazardous alcohol consumption } \\
\hline No & $95(59)$ & $65(41)$ & 1.00 & - & & \\
\hline Yes & $19(68)$ & $9(32)$ & $0.69(0.29-1.63)$ & 0.398 & & \\
\hline \multicolumn{7}{|l|}{ Current OAT } \\
\hline No & $44(65)$ & $24(35)$ & 1.00 & - & & \\
\hline Yes & $69(58)$ & $51(43)$ & $1.36(0.73-2.51)$ & 0.333 & & \\
\hline \multicolumn{7}{|c|}{ Injecting (last month) $)^{\mathrm{a}}$} \\
\hline No & $51(70)$ & $22(30)$ & 1.00 & - & & \\
\hline Yes & $63(55)$ & $52(45)$ & $1.91(1.03-3.56)$ & 0.040 & & \\
\hline \multicolumn{7}{|c|}{ Frequency of injecting (last month) ${ }^{\mathrm{a}}$} \\
\hline Never & $51(70)$ & $22(30)$ & 1.00 & - & & \\
\hline Less than daily & $40(53)$ & $35(47)$ & $2.03(1.03-3.98)$ & 0.040 & & \\
\hline Daily or greater & $23(58)$ & $17(43)$ & $1.71(0.77-3.82)$ & 0.188 & & \\
\hline \multicolumn{7}{|c|}{ Any injecting during treatment } \\
\hline No & $42(68)$ & $20(32)$ & 1.00 & - & & \\
\hline Yes & $72(58)$ & $52(42)$ & $1.52(0.80-2.88)$ & 0.203 & & \\
\hline \multicolumn{7}{|c|}{ Heroin injecting (last month) } \\
\hline No & $65(61)$ & $41(39)$ & 1.00 & - & & \\
\hline Yes & $48(59)$ & $34(41)$ & $1.12(0.62-2.02)$ & 0.699 & & \\
\hline \multicolumn{7}{|c|}{ Cocaine injecting (last month) } \\
\hline No & $102(62)$ & $62(38)$ & 1.00 & - & & \\
\hline Yes & $11(50)$ & $11(50)$ & $1.65(0.67-4.02)$ & 0.275 & & \\
\hline \multicolumn{7}{|c|}{ Amphetamine injecting (last month) } \\
\hline No & $92(66)$ & $48(34)$ & 1.00 & - & & \\
\hline Yes & $21(46)$ & $25(54)$ & $2.28(1.16-4.49)$ & 0.017 & & \\
\hline \multicolumn{7}{|c|}{ Cocaine/amphetamine injecting (last month) } \\
\hline No & $82(66)$ & $42(34)$ & 1.00 & - & & \\
\hline Yes & $31(48)$ & $33(52)$ & $2.08(1.12-3.85)$ & 0.020 & $2.48(1.28-4.82)$ & 0.007 \\
\hline \multicolumn{7}{|l|}{ Fibrosis stage } \\
\hline $\mathrm{F} 0-\mathrm{F} 1$ & $77(61)$ & $50(39)$ & 1.00 & - & & \\
\hline $\mathrm{F} 2-\mathrm{F} 3$ & $23(59)$ & $16(41)$ & $1.07(0.52-2.22)$ & 0.853 & & \\
\hline $\mathrm{F} 4$ & $10(59)$ & $7(41)$ & $1.08(0.39-3.02)$ & 0.886 & & \\
\hline \multicolumn{7}{|l|}{ Dosing pattern } \\
\hline Once-daily & $71(69)$ & $32(31)$ & 1.00 & - & & \\
\hline Twice-daily & $43(49)$ & $44(51)$ & $2.27(1.26-4.11)$ & 0.007 & $2.81(1.47-5.36)$ & 0.002 \\
\hline
\end{tabular}

${ }^{a}$ Not included in adjusted analysis due to collinearity with drug type. 


\section{FIGURE LEGENDS}

Figure 1: OAT and injecting status of participants from SIMPLIFY (blue) and D3FEAT (orange) at enrolment.

Figure 2: Blister packs used for dosing and adherence monitoring in DEFEAT (A) and SIMPLIFY (B).

Figure 3: Daily adherence to therapy in SIMPLIFY (top) and D3FEAT (bottom) measured by weekly administered electronic blister packs. Each row represents an individual patient and each column represents one day of therapy. Dark blue boxes represent $100 \%$ of prescribed doses received, midblue represents 50\% of daily doses received (in D3FEAT only) and light blue boxes represent no dose received. White boxes represent early discontinuation of treatment. Failure to achieve SVR due to virologic failure (red), reinfection (yellow), loss to follow-up (grey), and death (black) is denoted on the right.

Figure 4: Violin plot of adherence to HCV DAA treatment among people receiving once-daily therapy (blue) and twice-daily therapy (orange).

Figure 5: Mean daily adherence over the course of therapy among those receiving once-daily (blue) and twice-daily (orange) dosing. 
Figure 1.

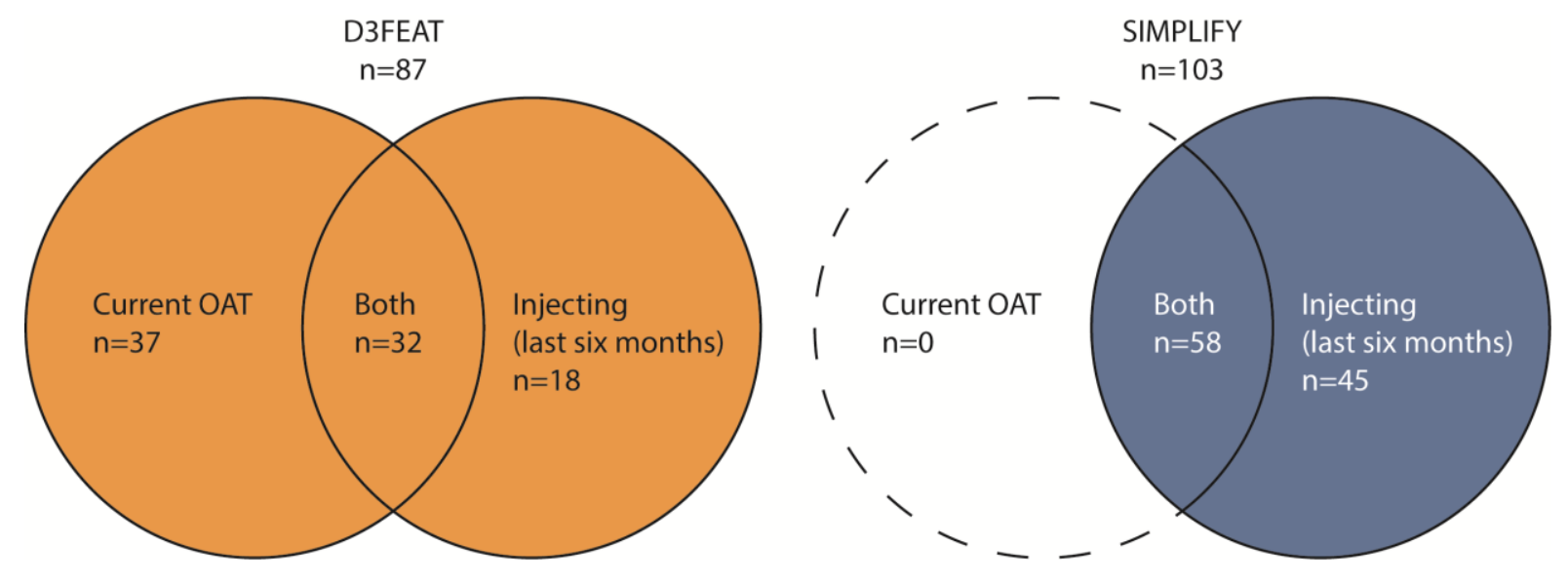


Figure 2.

A)

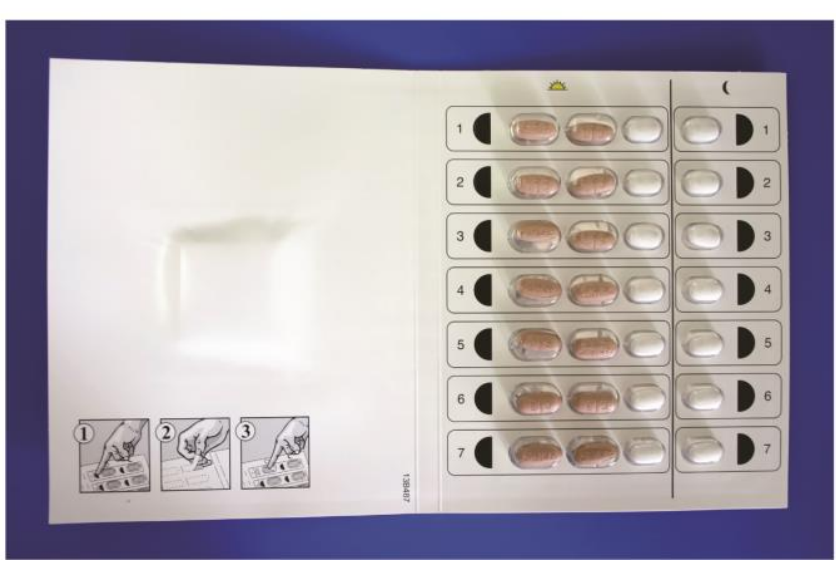

B)

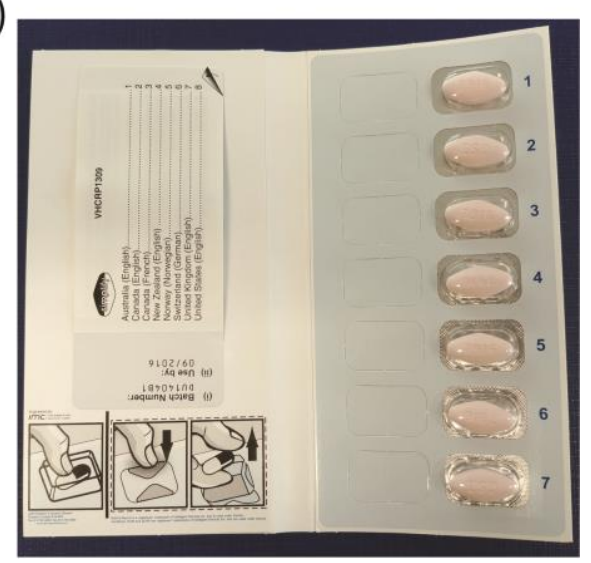


Figure 3.

Treatment days
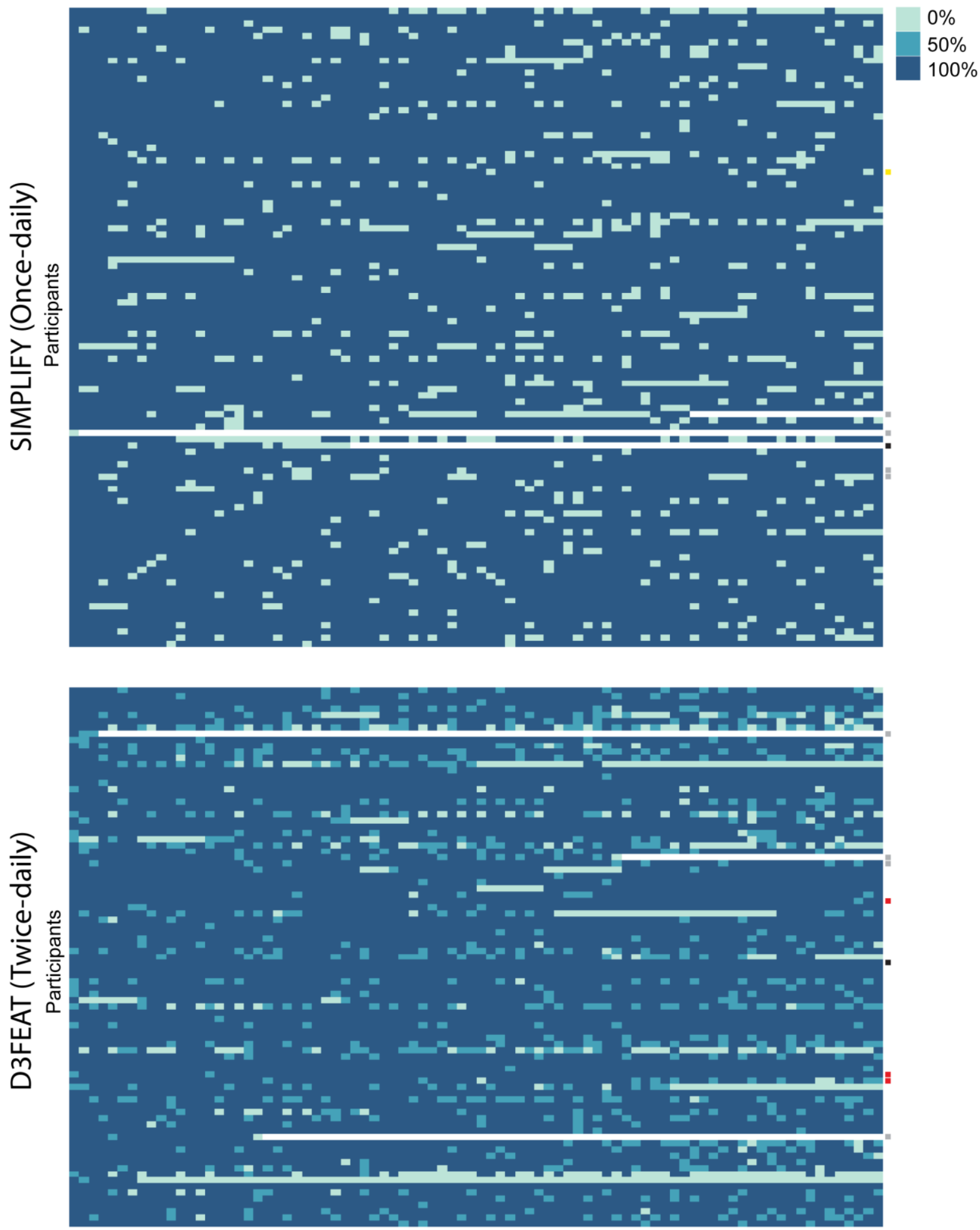
Figure 4.

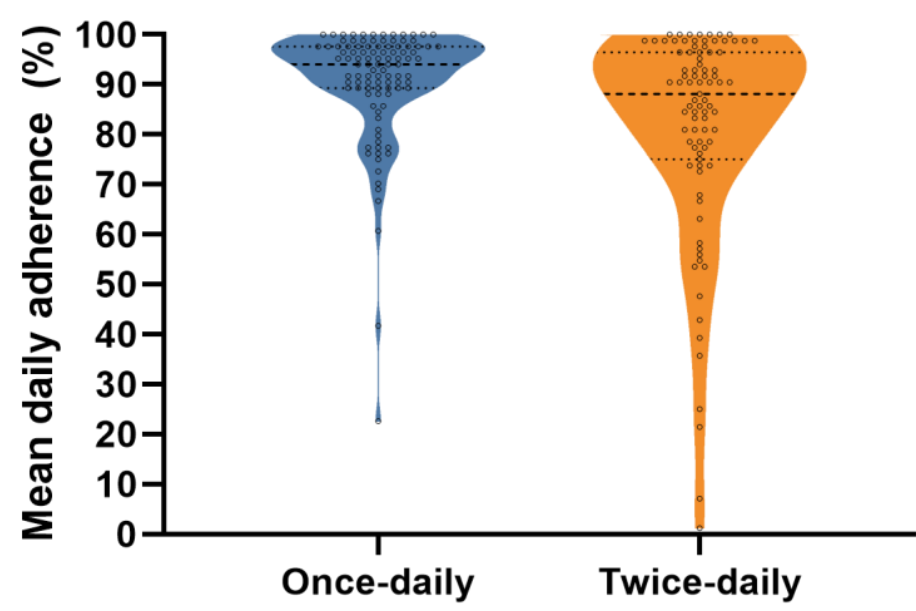


Figure 5.

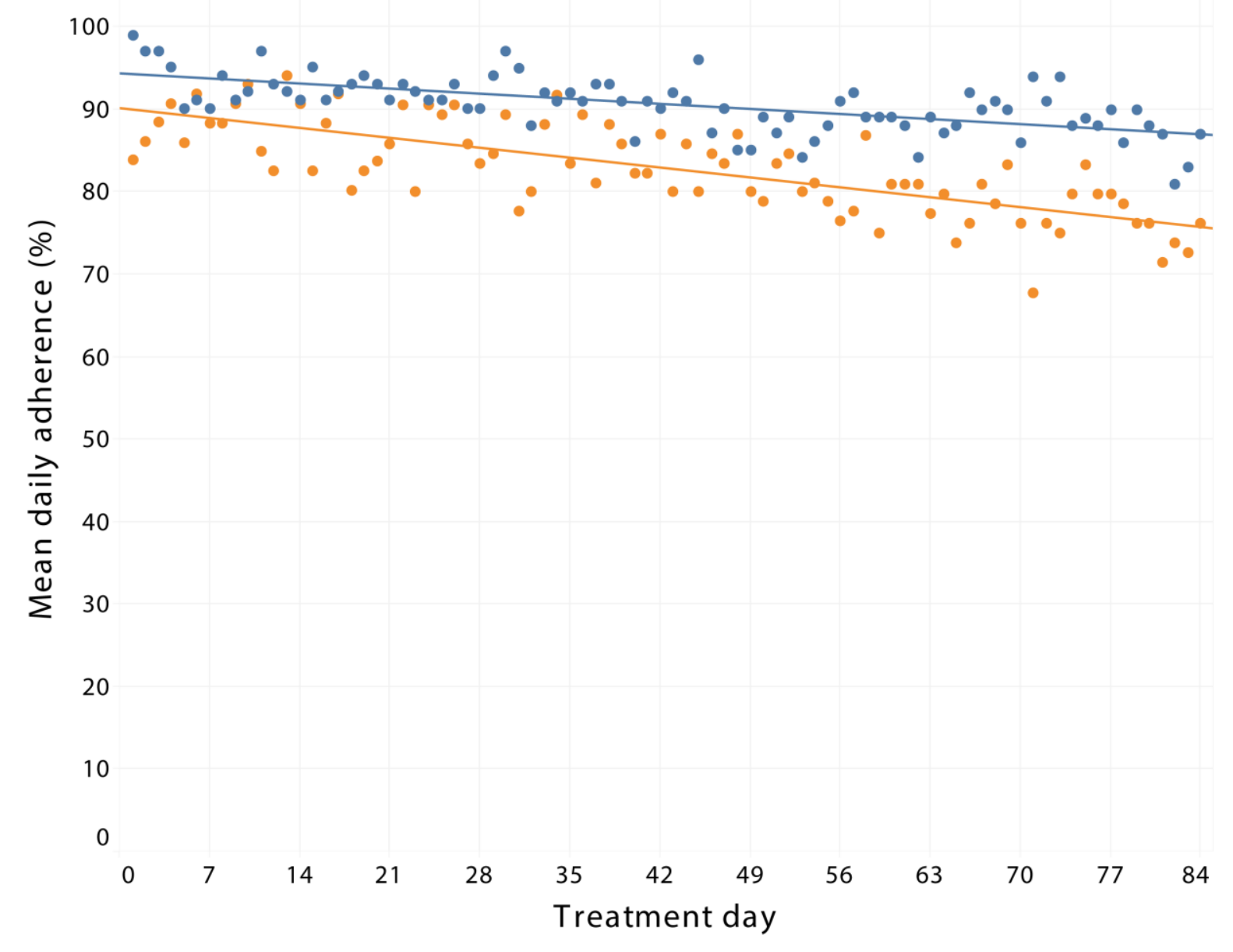

